\title{
Translating materials design to the clinic
}

\author{
Jeffrey A. Hubbell and Robert Langer
}

\begin{abstract}
Many materials-based therapeutic systems have reached the clinic or are in clinical trials. Here we describe materials design principles and the construction of delivery vehicles, as well as their adaptation and evaluation for human use.
\end{abstract}

M aterials-based therapeutics are having substantial impact on clinical medicine, for example in cancer therapy, vaccine development, and tissue repair and regeneration, fuelled by the rich innovation in fundamental science and the development of a host of technological approaches. In this Commentary, we highlight exciting advances in materials science, mostly applied to drug delivery, which are approaching the reality of clinical translation. We discuss examples from the early stages of the drug-discovery pipeline to those well on the road to clinical approval. We describe materials design from concept to demonstration in rodent models of disease, to the adaptation and evaluation for therapeutic use in patients.

For illustrative purposes and in the interest of brevity, we discuss selected examples only. Broader coverage in the areas of nanomaterials ${ }^{1-3}$ and cancer therapeutics ${ }^{4,5}$ can be found elsewhere.

\section{Tumour vascular targeting}

The motivation for pursuing nanomaterials in cancer therapeutics has long been the higher permeability to colloids of tumour vasculature relative to healthy vasculature - the so-called enhanced permeability and retention (EPR) effect ${ }^{1}$. Whereas small molecules may permeate equally well within healthy and tumourassociated vasculature, nanoscale materials permeate much more readily through the tumour vasculature, providing a means for tumour-specific targeting based on the physiology of the tumour ${ }^{6,7}$. Below we highlight selected examples of materials in the sub-100-nm size range that can exploit the EPR effect for tumour targeting.

One anticancer delivery vehicle that may be clinically successful incorporates paclitaxel (a hydrophobic drug) within the hydrophobic cores of micelles formed from amphiphilic block copolymers comprising poly(ethylene glycol) (PEG) and chemically modified poly (aspartic acid) ${ }^{8}$. The size of the micelles and the critical micelle concentration can be controlled by manipulating the degree of polymerization of the modified poly(aspartic acid), and a spherical morphology can be obtained by selecting PEG of an appropriate degree of polymerization. The hydrophobicity of the drug cargo drives it into the hydrophobic micellar core, and thus ensures drug solubilization. Release from the nanoscale system is provided by slow dissolution into the aqueous biological fluids. The translation of this paclitaxel-incorporating polymeric delivery system, namely NK105, into the clinic is being led by NanoCarrier. The micellar nanomaterials are about $85 \mathrm{~nm}$ in diameter ${ }^{9}$, and have shown increased bioavailability (as measured by plasma area under the curve (AUC) values or as a measure of time-integrated drug exposure) of $>50$-fold in rodents ${ }^{8}$ and $>15$-fold in phase I clinical studies ${ }^{9}$ compared with the free drug. NK105 has also demonstrated markedly reduced nephrotoxicity in animal models. A limited number of phase II studies have been published ${ }^{10}$, and the path of NK105 towards clinical reality remains to be seen. In the design of NK105, micelles in the sub-100-nm size range were produced to allow extravasation in the hyperpermeable tumour vasculature, and hydrophobic domains were introduced into the nanomaterials to solubilize hydrophobic drugs.

Investigation of the EPR effect using animal models has revealed that the vasculature of some tumours allowed extravasation of micelles around $100 \mathrm{~nm}$, but that the vasculature of other tumour types was less hyperpermeable. Such variability is also thought to exist in human tumours of different types ${ }^{7}$. When smaller micelles were tested, those with a 30-nm diameter were observed to penetrate the vasculature of all tested tumours in the animal models ${ }^{11}$, thus revealing that very small nanoparticles are more useful than larger ones. Clinical results from a second anticancer micellar delivery system currently under development by NanoCarrier are consistent with this observation. This delivery system, NC-6004, is formed from a block copolymer of PEG and poly(glutamic acid). Complexation of cisplatin to the carboxyl side-chains of the copolymer generates 30 -nm-diameter micelles, which are in the advantageous size regime ${ }^{12}$. When these cisplatin-loaded micelles were evaluated in a rodent model, the drug displayed lower neurotoxicity and nephrotoxicity than the free drug, as well as a 65 -fold enhancement in $\mathrm{AUC}^{13}$. In a phase I clinical investigation, more than an 8 -fold enhancement in AUC was observed ${ }^{14}$, indicating a reduction in off-target toxicity. Thus, in its design, NC-6004 has the ability to harness the EPR effect as well as use chemical complexation to provide drug loading.

\section{We hope that research will further connect observations in animal models to the elucidation of efficacy and safety in human clinical applications.}

More refined micellar designs are also being pursued. For example, polymer micelles with complexed drugs have been designed to release their payload only in acidic endosomal compartments, which are physically located near the cell's nucleus ${ }^{15}$. Specifically, the complexation of an oxaliplatin compound to a PEG- 
poly(glutamic acid)-based block copolymer has been shown to deliver the drug to the nucleus in a sufficiently targeted manner to avoid detoxification mechanisms that are present in the cytoplasm and can lead to drug resistance. Given that the chemistry of these micelles is similar to that of NC-6004 this design should also be translatable to the clinic.

The ability to design polymeric nanoparticles that can also achieve cellular targeting may be an even more effective strategy. Nanoparticles composed of Food and Drug Administration-approved materials (such as copolymers of lactic and glycolic acid) that have an outer PEG shell circulate for a long time in animals ${ }^{16}$, and can be directed towards tumours by adding a peptide to the nanoparticles' outer surface that targets receptors that are overexpressed in tumour vasculature. Initial studies by BIND Therapeutics have shown these hybrid materials to have an acceptable safety profile and to be a potentially effective approach for targeting solid tumours in humans ${ }^{17}$. The polymeric nanoparticle formulation BIND-014, which is in phase II clinical trials, entraps the chemotherapeutic drug
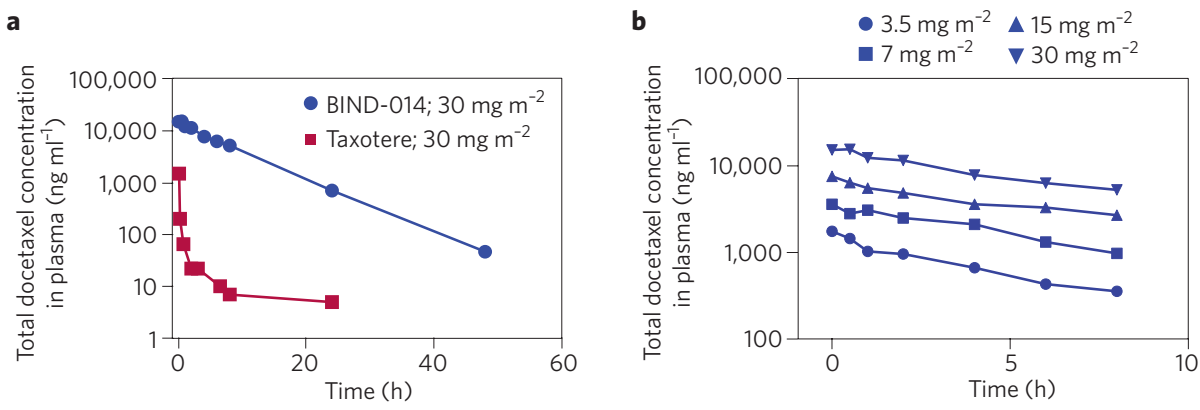

c
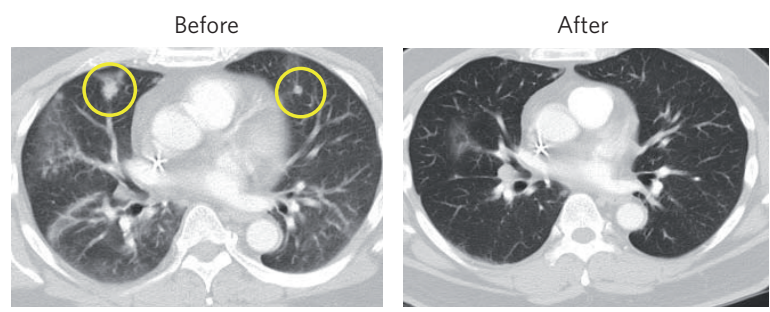

Figure 1 | A chemotherapeutic drug docetaxel entrapped within a 100-nm-diameter nanoparticle made from block-copolymers of polylactic acid and PEG is currently in a phase II clinical trial (being carried out by BIND Therapeutics) ${ }^{17}$. The nanoparticles are functionalized with a small-molecule ligand for targeting the prostate-specific membrane antigen (PSMA). Studies in animal models demonstrate that the nanoparticles find their target by means of the EPR effect and the ligand-mediated biomolecular interactions. a, Phase I studies demonstrate that the pharmacokinetics of the drug in humans is improved by entrapping it within nanoparticles, with slower clearance from blood plasma and at least a 100fold elevated increase in plasma levels compared with the free form of the drug (Taxotere, a clinical formulation of docetaxel). b. These clinical studies also show that the level of the drug in plasma can be predicted from the injected dose. c, Disappearance or shrinkage of the metastases in a patient with multiple lung metastases (circled in yellow) of cholangiocarcinoma was observed. Figure reproduced with permission from ref. 17, (c) 2012 AAAS.
In addition to the tumour cells themselves being a target, delivery to other cells in the tumour may be equally powerful. Recent studies in mouse models report a nanoscale delivery system that homes to the tumour vasculature by targeting coagulum present in the vessels ${ }^{18}$, reducing blood flow and thus nutrition to the tumour. Additionally, a recent study with docetaxel covalently linked to 120-nm-diameter nanoparticles showed a substantial effect on tumour stromal cells ${ }^{19}$, which provide supporting and immune regulatory function in the tumour and modulate tumour vascular permeability. It may be that these nanoparticles have some unknown special feature that targets stroma, or that the many nanoparticle formulations that penetrate into the tumour deliver drug more efficiently to the stroma compared with injection of the free drug.

\section{Delivery of small interfering RNA}

The anticancer therapies we have discussed all involve hydrophobic drugs for which solubility is a problem, however, their permeation of hydrophobic cell membranes is straightforward. Therapeutic molecules that are hydrophilic, such as small interfering RNA (siRNA) ${ }^{20,21}$, however, behave in the opposite manner. Indeed, siRNA is highly soluble, but its hydrophilicity restricts membrane permeation and access to the cytoplasm, where the drug is active. These hurdles can, nevertheless, be overcome through materials design. For example, because siRNA is negatively charged, researchers have developed nanoparticle-forming cationic polymers to deliver siRNA to the cytoplasm $^{22}$. More specifically, a watersoluble polymer made of an oligomer of cyclodextrin (a sugar-based macrocycle with a hydrophobic 'host' core) can be chemically modified to become cationic on its exterior, allowing electrostatic complexation with siRNA to form sub100-nm nanoparticles (Fig. 2). From other studies, however, it has been shown that cationic amino-functionalized polymers can cause disruption of endosomes after cellular uptake because of the protonsponge effect ${ }^{23}$ - that is, as protons are pumped into the endosome during the normal process of endosomal acidification, the amine functions neutralize the charge, which leads to an osmotic imbalance and rupture of the endosome. As a consequence of their positive charge, the cyclodextrinbased nanoparticles can undergo nonspecific clearance; however, this can be blocked by attaching hydrophobic tags to the termini of hydrophilic and 
nonionic PEG chains, forming host-guest interactions with the cyclodextrin core (Fig. 2). Furthermore, because the transferrin receptor is upregulated in many tumours, transferrin can be attached to the PEG host to target tumour cells by molecular recognition ${ }^{24}$. In a mouse model, transferrin targeting led to a doubling of tumour-specific knockdown of gene expression ${ }^{24}$ compared with that achieved with the EPR effect alone. Thus, this macrocyclic siRNA delivery system is an example of a combination of design principles: electrostatic complexation for drug loading, the induction of an osmotic imbalance for promoting drug permeation into the cytoplasm, and the molecular targeting of biomolecular features specific to tumour cells that lie beneath the hyperpermeable vasculature.

This cyclodextrin-based targeted siRNA delivery system is in clinical development at Arrowhead Research Corporation. The product, CALAA-01, uses an siRNA sequence to block expression of the M2 subunit of ribonucleotide reductase. CALAA-01 has been characterized in animal models ${ }^{25}$ and in human clinical studies, and has shown tumourspecific knockdown in patients with metastatic melanoma ${ }^{26}$.

Another approach for siRNA delivery involves its encapsulation in liposomes a strategy that is particularly attractive for targeting the liver because of the organ's role in particle clearance. Although liposomes have been used for some 50 years, the number of lipids that have been used for creating them has been very limited. Recently, a high-throughput synthesis approach has made it possible to generate and screen thousands of new lipids ${ }^{27-29}$, enabling the creation of liposome-like structures that have progressively lowered the amount of siRNA that is required to achieve knockdown of genes by three or four orders of magnitude. Molecularly self-assembled nucleic acid nanoparticles with precisely controlled origami structures have also been synthesized for targeted siRNA delivery ${ }^{30}$.

\section{Responsive nanomaterials}

Phase transitions in nanomaterials are being used to address the issue of poor penetration of hydrophilic drugs through cellular membranes. After endocytosis from the extracellular milieu, the endosome is slowly acidified from $\mathrm{pH} 7.4$ to approximately $\mathrm{pH} 6.5$ in the early endosome, to $\mathrm{pH} 6.0$ in the late endosome and eventually to approximately pH 5.5 after fusion with the lysosome ${ }^{31}$. In the context of sensitive biomolecular
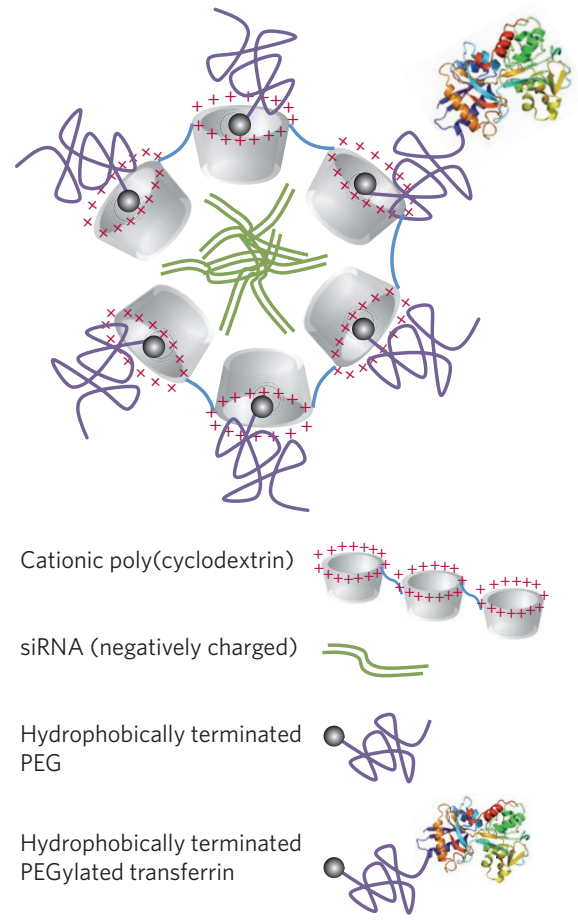

Figure 2 | In a phase I study in cancer patients an siRNA formulation CALAA-01 demonstrated efficient knockdown of gene expression in human tumours. The siRNA nanoparticle is based on a polymer of cationically derivatized cyclodextrin complexed with the anionic siRNA payload. The nanoparticles are stabilized by binding of hydrophobically terminated PEG to the cyclodextrin rings, which occurs via the interaction of the hydrophobe (guest) within the core of the cyclodextrin (host). Delivery of cyclodextrinbound-PEG further grafted with transferrin - a protein for which many tumour cells over-express a receptor - resulted in knockdown of target messenger RNA and the reduction of protein levels in tumours, opening the door for further clinical investigations focused on antitumour activity ${ }^{24,26}$.

drugs, the earlier a drug can be induced to escape this path (which also involves exposure to enzymes and a harsh redox environment), the less degradation it should experience. Families of polymers have thus been designed with very sharp transitions in hydrophobicity as a function of $\mathrm{pH}^{32,33}$. These polymers do not affect the plasma membrane at the exterior surface of the cell ( $\mathrm{pH}$ 7.4), but after endocytosis and acidification to $\mathrm{pH} 6.5$ they disrupt the endosome, which leads to efficient cytoplasmic delivery of an otherwise membrane-impermeable payload. A few such polymers, under development by PhaseRx, have been shown to be useful for the delivery of sensitive hydrophilic macromolecules, including siRNA ${ }^{34}$ and plasmid DNA ${ }^{35}$. These materials exemplify a design principle for transporting hydrophilic drugs across biological membranes: engineering phase transitions to hydrophobic, membrane-destabilizing structures, but only at $\mathrm{pH}$ values encountered after cellular uptake.

The drug-carrying self-assembling micelles discussed so far are made from synthetic polymers. However, researchers have demonstrated that recombinant proteins can also be used. Some proteins have evolved a thermoresponsive self-assembling character, with elastin being a well-studied example. Elastin comprises a pentapeptide repeat, VPGVG, which self-assembles to form a gel-like state $^{36}$. The sequence can be generalized to VPGXG, where $\mathrm{X}$ can be any amino acid except proline. The biophysical properties of the amino acid X in such elastin-like peptides (ELPs) ${ }^{36}$ affect the properties of the resulting gel, including its sol-gel transition temperature. In one implementation, a recombinant protein block-copolymer with a soluble ELP fused to a polypeptide domain rich in cysteine was designed so that the cysteine could be used to conjugate the chemotherapeutic, doxorubicin, the hydrophobicity of which drove the formation of sub-100-nm micelles $^{37}$. The doxorubicin-loaded micelles targeted the tumour vasculature by means of the EPR effect, and led to enhanced tumour killing and reduced systemic toxicity of the drug in tumour models ${ }^{37}$. In a more sophisticated implementation, the thermal sensitivity of ELP-based protein-drug conjugates was exploited for combined chemotherapy and hyperthermia therapy. In this case, heat applied to the tumour was used to destabilize the polymer micelles, leading to the increase of small-molecule drug deposition in the tumour ${ }^{38}$. By carefully adjusting the amino acid sequence of the ELP as well as its molecular weight and degree of drug grafting, thermal transitions between $37^{\circ} \mathrm{C}$ and $42^{\circ} \mathrm{C}$ could be achieved, which permitted hyperthermic tumour-specific targeting at modest temperature changes. In these ELP-based drugs, we identify the design principles of self-assembly by biomimicry and of triggering drug release by external manipulation, and, more specifically, of using localized heating to drive the nanomaterial through a thermal phase transition.

The translation of the design principle of ELP self-assembly to the clinic can also be achieved by using recombinant protein drugs. Given that the proteins are produced by a recombinant methodology, recombinant fusions of therapeutic proteins with ELP domains could yield protein 
variants that self-assemble at the site of injection to form a depot ${ }^{39}$. Moreover, because such an ELP assembly occurs in a dynamic manner and ELPs can dissociate into soluble unimers comprising the ELP domain fused to the bioactive protein domain, the therapeutic proteins in the depot should slowly escape into systemic circulation. PhaseBio Pharmaceuticals is developing an ELP-fused GLP-1 receptor agonist for type 2 diabetes. This design of a single recombinant fusion protein made of the drug and the self-assembling polymer allows the conjugate to be produced in a more precise and efficient manner than could otherwise be attained through chemical grafting of the protein to the polymer. In this example, the drugdelivery system combines the concepts of drug retention and drug release into its very structure.

\section{Nanomaterials for phototherapy}

Drug-delivery particles function as carriers of the active agent, yet in some cases the particle itself can be the active agent. For example, plasmonic gold nanoshells in the sub-50-nm regime are known to efficiently absorb near-infrared light and subsequently produce heat ${ }^{40-42}$. Hyperthermia at the site of vascular permeability has been shown to lead to selective and efficient photothermal killing of tumours (when irradiated with a near-infrared laser, either directly or from a fibre optic probe) in animal models as a consequence of the accumulation of the nanoshell at tumour sites because of the EPR effect ${ }^{43}$. These materials, referred to as AuroShell particles, are being tested in the clinic by NanoSpectra Biosciences. Interestingly, because there is no bioactive molecule involved in the therapy, AuroShell particles are regulated as a medical device rather than a drug. As well as taking advantage of the EPR effect, tumour targeting can also be achieved by means of nanoparticle-associated antibodies that bind to HER2 on tumour cells ${ }^{44,45}$ or to VEGF-R2 on sites of active angiogenesis in tumours ${ }^{46}$.

\section{Outlook}

We have illustrated several materials and some physiological and biophysical design principles - the EPR effect, self-assembly, controlled dissociation, phase transitions and disruption of biological barriers - and discussed how they can be leveraged and interconnected to achieve drug-delivery efficacy in disease models. Moreover, we have highlighted a few therapeutic areas where materials may play an important role in the future of medicine, but numerous challenges remain.

We hope that research will further connect observations in animal models to the elucidation of efficacy and safety in human clinical applications. More specifically, future approaches may enable drugs to be delivered by sending radiofrequency waves activated by remote control to microchips implanted in the human body ${ }^{47}$. New tissues and organs that are created using a combination of materials and cells may revolutionize transplantation, perhaps enabling the production of drugs in situ using cells or the generation of functional organs. Examples include nanowires being put into engineered heart tissue to provide enhanced performance ${ }^{48}$ and nanowired sensors being placed into a variety of tissues to monitor and improve tissue function ${ }^{49}$. Creating new materials that resist bacterial adhesion ${ }^{50}$ and prevent thrombus formation when in contact with blood ${ }^{51}$ is also extremely important. Nanomaterials are also impacting the field of vaccines, for example, by using interstitial flow to direct very small nanoparticles to dendritic cells resident in the lymph nodes ${ }^{52-55}$. The next years of clinical development will certainly be exciting for materials-based drug-delivery therapeutics as well as for materials-based tissue engineering and medical devices.

Jeffrey A. Hubbell is at the Institute for Bioengineering, School of Life Sciences and School of Engineering, and Institute for Chemical Sciences and Engineering, School of Basic Sciences, Ecole Polytechnique Fédérale de Lausanne (EPFL), CH-1015 Lausanne, Switzerland. Robert Langer is at the Department of Chemical Engineering and Koch Institute for Integrative Cancer Research, Massachusetts Institute of Technology, Cambridge, Massachusetts 02139, USA.

e-mail: jeffrey.hubbell@epfl.ch; rlanger@mit.edu

\section{References}

1. Hubbell, J. A. \& Chilkoti, A. Science 337, 303-305 (2012).

2. Petros, R. A. \& DeSimone, J. M. Nature Rev. Drug Discov. 9, 615-627 (2010).

3. Yoo, J. W., Irvine, D. J., Discher, D. E. \& Mitragotri, S. Nature Rev. Drug Discov. 10, 521-535 (2011)

4. Davis, M. E., Chen, Z. G. \& Shin, D. M. Nature Rev. Drug Discov. 7, 771-782 (2008).

5. Wang, A. Z., Langer, R. \& Farokhzad, O. C. Annu. Rev. Med. 63, 185-198 (2012)

6. Baish, J. W. et al. Proc. Natl Acad. Sci. USA 108, 1799-1803 (2011).
7. Prabhakar, U. et al. Cancer Res. 73, 2412-2417 (2013).

8. Hamaguchi, T. et al. Br. J. Cancer 92, 1240-1246 (2005)

9. Hamaguchi, T. et al. Br. J. Cancer 97, 170-176 (2007).

10. Kato, K. et al. Invest. New Drugs 30, 1621-1627 (2012).

11. Cabral, H. et al. Nature Nanotech. 6, 815-823 (2011).

12. Endo, K. et al. Cancer Sci. 104, 369-374 (2013).

13. Uchino, H. et al. Br. J. Cancer 93, 678-687 (2005).

14. Plummer, R. et al. Br. J. Cancer 104, 593-598 (2011).

15. Murakami, M. et al. Sci. Transl. Med. 3, 64ra62 (2011).

16. Gref, R. et al. Science 263, 1600-1603 (1994).

17. Hrkach, J. et al. Sci. Transl. Med. 4, $128 \mathrm{ra1} 39$ (2012).

18. Von Maltzahn, G. et al. Nature Mater. 10, 545-552 (2011)

19. Murakami, M. et al. Cancer Res. 73, 4862-4871 (2013).

20. Castanotto, D. \& Rossi, J. J. Nature 457, 426-433 (2009).

21. De Fougerolles, A., Vornlocher, H. P., Maraganore, J. \&

Lieberman, J. Nature Rev. Drug Discov. 6, 443-453 (2007).

22. Davis, M. E. Mol. Pharm. 6, 659-668 (2009)

23. Boussif, O. et al. Proc. Natl Acad. Sci. USA 92, 7297-7301 (1995).

24. Bartlett, D. W., Su, H., Hildebrandt, I. J., Weber, W. A. \& Davis, M. E. Proc. Natl Acad. Sci. USA 104, 15549-15554 (2007).

25. Rahman, M. A. et al. J. Control. Release 159, 384-392 (2012).

26. Davis, M. E. et al. Nature 464, 1067-1070 (2010).

27. Akinc, A. et al. Nature Biotechnol. 26, 561-569 (2008).

28. Love, K. T. et al. Proc. Natl Acad. Sci. USA 107, 1864-1869 (2010).

29. Sahay, G. et al. Nature Biotechnol. 31, 653-658 (2013).

30. Lee, H. et al. Nature Nanotech. 7, 389-393 (2012).

31. Padilla-Parra, S. et al. Proc Natl Acad Sci USA 109, 17627-17632 (2012).

32. Convertine, A. J., Benoit, D. S., Duvall, C. L., Hoffman, A. S. \& Stayton, P. S. J. Control. Release 133, 221-229 (2009).

33. Yin, X., Hoffman, A. S. \& Stayton, P. S. Biomacromolecules 7, 1381-1385 (2006)

34. Lundy, B. B., Convertine, A., Miteva, M. \& Stayton, P. S. Bioconjug. Chem. 24, 398-407 (2013)

35. Manganiello, M. J., Cheng, C., Convertine, A. J., Bryers, J. D. \& Stayton, P. S. Biomaterials 33, 2301-2309 (2012).

36. McDaniel, J. R. et al. Angew. Chem. Int. Ed. 52, 1683-1687 (2013).

37. MacKay, J. A. et al. Nature Mater. 8, 993-999 (2009).

38. McDaniel, J. R., Macewan, S. R., Dewhirst, M. \& Chilkoti, A. J. Control. Release 159, 362-367 (2012)

39. Hassouneh, W., MacEwan, S. R. \& Chilkoti, A. Methods Enzymol. 502, 215-237 (2012)

40. Hirsch, L. R. et al. Proc. Natl Acad. Sci. USA 100, 13549-13554 (2003).

41. Kennedy, L. C. et al. Small 7, 169-183 (2011).

42. O’Neal, D. P., Hirsch, L. R., Halas, N. J., Payne, J. D. \& West, J. L. Cancer Lett. 209, 171-176 (2004).

43. Gobin, A. M., Watkins, E. M., Quevedo, E., Colvin, V. L. \& West, J. L. Small 6, 745-752 (2010).

44. Bernardi, R. J., Lowery, A. R., Thompson, P. A., Blaney, S. M. \& West, J. L. J. Neurooncol. 86, 165-172 (2008).

45. Day, E. S. et al. Int. J. Nanomedicine 5, 445-454 (2010).

46. Day, E. S. et al. Nanomedicine 7, 1133-1148 (2012).

47. Farra, R. et al. Sci. Transl. Med. 4, 122ra121 (2012).

48. Dvir, T. et al. Nature Nanotech. 6, 720-725 (2011)

49. Tian, B. et al. Nature Mater. 11, 986-994 (2012).

50. Hook, A. L. et al. Nature Biotechnol. 30, 868-875 (2012).

51. Smith, R. S. et al. Sci. Transl. Med. 4, 153ral32 (2012).

52. Kourtis, I. C. et al. PLoS ONE 8, e61646 (2013).

53. Moon, J. J. et al. Nature Mater. 10, 243-251 (2011).

54. Nembrini, C. et al. Proc. Natl Acad. Sci. USA 108, E989-E997 (2011).

55. Reddy, S. T. et al. Nature Biotechnol. 25, 1159-1164 (2007).

\section{Acknowledgements}

J.A.H. thanks the Swiss National Science Foundation and the European Research Council for grant support, and R.L. thanks the National Institute for Health and the National Cancer Institute for grant support.

Competing financial interests

R.L. is a founder and shareholder of BIND Therapeutics, Semprus, and Microchips, and a member of the scientific advisory board and shareholder of Alnylam. 